\title{
PERCEPCIÓN DE PERSONAS MAYORES SOBRE LA ATENCIÓN RECIBIDA EN INSTITUCIONES DE SALUD DE LA CIUDAD DE DURANGO
}

\author{
PERCEPTION BY THE ELDERLY REGARDING CARE \\ IN HEALTH INSTITUTIONS IN DURANGO CITY \\ María Concepción Arroyo Rueda \\ Universidad Juárez del Estado de Durango \\ aguaconflores@hotmail.com \\ Liliana Vázquez García \\ Universidad Autónoma de Nuevo León \\ lilianita_vazquez@hotmail.com
}

\section{RESUMEN}

En este artículo se muestra la percepción de personas adultas mayores acerca de la atención que reciben en distintas instituciones del sector salud en la ciudad de Durango. Se utilizó una metodología de tipo cualitativo y entrevistas abiertas para recolectar los datos. Tras un muestreo de tipo intencional, algunos participantes fueron interrogados al salir de las instituciones de salud y otros en sus hogares respectivos. Los resultados muestran que, en promedio, la mitad califican de satisfactoria la atención que reciben, mientras que el resto tiene opiniones negativas. Esta última percepción gira en torno a un trato inadecuado por parte de los médicos, así como a largos periodos de espera y a escasez de medicamentos.

PALABRAS CLAVE: percepción, personas mayores, atención de salud.

\section{ABSTRACT}

This article demonstrates the perception that elderly people have regarding the care they receive in different healthcare institutions in Durango City. A qualitative methodology and open interviews were used for this data collection. After an intentional sampling, some participants were interviewed when leaving the healthcare institutions and others in their own homes. Results demonstrated that on average half of the participants held a negative opinion. This negative perception revolves around the inappropriate treatment provided by doctors, as well as lengthy waiting periods and lack of medication.

KEY WORDS: perception, the elderly, healthcare. 


\section{INTRODUCCIÓN}

L

a salud, uno de los bienes más preciados, constituye un derecho en todos los países. En México, el artículo $4^{\circ}$ de la Constitución dice a la letra: «toda persona tiene derecho a la protección de la salud. La ley definirá las bases y modalidades para el acceso a los servicios de salud y establecerá la concurrencia de la federación y las entidades federativas en materia de salubridad general». ${ }^{1}$

La salud es un tema de preocupación de los gobiernos y de la sociedad, sin embargo, aún se observa falta de cobertura, de accesibilidad, de disponibilidad y de calidad en la atención en muchas de las instituciones sanitarias. Como bien sabemos, México es un país de enormes desigualdades, y lo referente a la salud no es la excepción (Román y Valencia 2012, Juárez et al. 2014). En el tema de las personas adultas mayores y su acceso a la salud existe legislación específica y normas que garantizan este derecho para este grupo de población; ${ }^{2}$ sin embargo, en la realidad esto no se cumple cabalmente. El impacto de las desigualdades en el tema de la salud cobra relevancia en la medida en que se dejan de otorgar los servicios a los que se tiene derecho, o bien, se ofrecen de manera segmentada e insuficiente, como es el caso de las poblaciones rurales o indígenas alejadas de la infraestructura urbana, si bien en el contexto (urbano) no se está exento de otro tipo de limitaciones.

Estudios realizados en el Instituto Nacional de Salud Pública han hecho importantes aportes sobre los retos y desafíos que enfrenta este sector de la población «considerado el más vulnerable del país» para atender sus necesidades de sanidad (Salgado 2007, Salgado et al. 2005, Ruelas y Salgado 2008). Otra contribución importante en este renglón es la del grupo de investigadores del Instituto Nacional de Geriatría (INGER), cuyos trabajos han girado en torno a la biología del envejecimiento, investigación clínica y epidemiológica, principalmente, y

\footnotetext{
${ }^{1}$ En México, el derecho a la salud forma parte de los derechos sociales, lo que obliga al Estado a garantizarlo y protegerlo ofreciendo una serie de prestaciones y medios necesarios para satisfacer las necesidades correspondientes; es decir, el Estado interviene por medio de políticas gubernamentales, programas sociales o reformas legales, con el objeto de que el derecho a la salud pueda ejercerse plenamente. Y aunque en materia de reglamentación el derecho a la salud en México está garantizado por la Constitución y alineado con reglamentaciones internacionales, esto no siempre implica su pleno ejercicio, por lo que es necesario conocer la brecha que existe entre ese reconocimiento del derecho y su ejercicio efectivo (CONEVAL 2014).

${ }^{2}$ Además de la Constitución Política de los Estados Unidos Mexicanos, la Ley para la Protección de las Personas Adultas Mayores plasma en el capítulo II, artículo V, fracción III, el derecho a la salud, la alimentación y la familia. De la misma forma, el acceso a la salud como derecho se plasma en otros documentos normativos tanto a nivel general como local, es decir, en los estados.
} 
en menor proporción sobre modelos de atención gerontológica y determinantes sociales del envejecimiento (López y Torres 2012, Gutiérrez y Kershenobich 2012), destacando la utilización de metodologías cuantitativas. Otros esfuerzos dignos de mención, de la Secretaría de Salud, son la Encuesta Nacional de Salud y Envejecimiento en México (ENASEM 2003, citada en Wong, Espinoza y Palloni 2007), la Encuesta de Salud y Bienestar en el Envejecimiento (SABE), en donde se reporta la situación de salud de los mayores en distintos estados del país (Albalá et al. 2005), y la Encuesta Nacional de Salud y Nutrición, que trae un importante apartado sobre la salud de las personas mayores (ENSANUT 2012). Asimismo, investigadores del Instituto Mexicano del Seguro Social también han hecho importantes contribuciones sobre el tema (Muñoz et al. 2004).

Por otro lado, hay un buen número de personas mayores sin acceso a servicios de salud por no haber tenido la oportunidad de estudiar, no haberse insertado en el empleo formal y, en consecuencia, no cuentan con seguridad social (que incluye la atención de la salud). Este fenómeno es más visible en el caso de las mujeres mayores, por su escasa o nula participación en el mercado laboral formal. Es así que la pobreza ${ }^{3}$ y la falta de acceso a los servicios de salud, sumadas a problemáticas de vivienda y alimentación ponen en permanente situación de riesgo la salud de las personas mayores, tal como se confirma en un estudio realizado por la Comisión Económica para América Latina (CEPAL), en cuyo reporte se dice que uno de cada tres adultos mayores no tiene acceso a la seguridad social y se encuentra en los cuatro deciles ${ }^{4}$ de más bajos ingresos, y tampoco son beneficiarios de ningún programa (Rubio y Garfias 2010).

En este sentido, la Organización Mundial de la Salud (2015) advierte que para combatir las desigualdades sociales es necesario que los países enfoquen sus políticas públicas en modificar los determinantes sociales de la salud, que diseñen políticas de salud en concordancia con las necesidades locales y teniendo en cuenta a los grupos sociales con mayores necesidades, a sabiendas de que cada vez más se incrementa el número de personas mayores y sus requerimientos de atención médica. Con esto no queremos afirmar que envejecimiento sea igual

\footnotetext{
${ }^{3}$ En 2012 había 10.9 millones de senescentes en México (INEGI 2013), de los cuales solo dos de cada 10 pueden solventar sus gastos y los ocho restantes viven en situación de pobreza (Promoción de la Salud y Determinantes Sociales 2013-2018).

${ }^{4}$ Un decil se utiliza principalmente para definir sectores socioeconómicos según ingreso familiar per cápita, es decir, según el total de dinero que aporta cada integrante de un hogar, dividido entre el número de sus miembros. Permite diferenciar a la población por nivel de ingreso según integrantes de la familia. Así, un decil representa a la población con la condición socioeconómica más vulnerable, y 10 deciles, a la población con mayores ingresos del país (Casen 2013).
} 
a enfermedad, pues los procesos de enfermedad no surgen por la vejez, sino que son producto de determinantes sociales que están presentes a lo largo de la vida. Lo que queremos puntualizar es que en la vejez, para unos más que para otros, estos procesos mórbidos se hacen presentes y demandan atención.

Las enfermedades más frecuentes en la población mayor reportadas por la Encuesta Nacional de Salud y Nutrición son la hipertensión, la diabetes, las enfermedades vasculares cerebrales y los tumores malignos (ENSANUT 2012). Todas exigen largos tratamientos y vigilancia periódica, y de ese modo generan altos costos y presiones financieras en las instituciones de salud (Promoción de la Salud y Determinantes Sociales 2013-2018).

Para combatir lo anterior, la política de salud vigente plantea los siguientes dos propósitos: 1. El abordaje de los determinantes sociales de la salud que contempla: educación y pobreza, infraestructura pública, infraestructura en el hogar; y 2. El acceso efectivo con calidad, que incluye: disponibilidad de oferta de servicios de salud en términos de cantidad y calidad relativas a las necesidades de la población; accesibilidad geográfica: distribución espacial de los servicios en relación a las necesidades de la población; accesibilidad económica: no existan barreras financieras de acceso; aceptabilidad: servicios de salud que se apeguen a las normas, las expectativas y las costumbres y creencias de la población (CONEVAL 2013).

Algunos de estos propósitos no se cumplen del todo pues, aunque hay una mayor cobertura, el gasto en medicina privada va en aumento respecto de la accesibilidad económica. Lo anterior puede ser un reflejo del acceso efectivo con calidad, ya que como se reporta en distintos trabajos, la calidad está fallando, principalmente en lo que se refiere a los tiempos de espera, abasto de medicamentos, relación médico-paciente, entre otros factores (Promoción de la Salud y Determinantes Sociales 2013-2018, Valdez et al. 2005, Campos et al. 2006). Si bien se ha mejorado el acceso a los servicios, los indicadores sobre oportunidad de la atención y acceso efectivo no han tenido una variación similar en tanto que un mayor número de personas cuenta con afiliación a estos (CONEVAL 2013), lo cual no garantiza que se esté recibiendo el servicio ni que, de ser así, este sea de calidad y resolutivo. Es conveniente entonces considerar elementos de análisis que permitan identificar si la cobertura se traduce en que, al demandarlos, los servicios sean otorgados y en qué medida son accesibles, de calidad y corresponden a las necesidades de las personas beneficiarias. 
El trabajo que presentamos busca conocer las percepciones de personas en edad avanzada en torno a su experiencia como usuarios de instituciones de salud y la atención que reciben. Explora también si esta última es satisfactoria o insatisfactoria en la medida en que se resuelven sus necesidades o si buscan alguna alternativa diferente. Para ello haremos una breve revisión de antecedentes, incluyendo algunas precisiones teórico-conceptuales mediante las cuales los resultados cobran sentido y significado.

\section{ANTECEDENTES DE LOS ESTUDIOS SOBRE SATISFACCIÓN EN LA ATENCIÓN A LA SALUD}

En este apartado hacemos una breve reseña de los estudios de la calidad de la atención médica. Si bien no es el propósito principal de este artículo profundizar sobre este aspecto, es conveniente dar a conocer los resultados más destacados de quienes han investigado sobre ello. Uno de los primeros en analizar este concepto fue Avedis Donabedian, quien establece cómo la evaluación del método y el enfoque dependerá de las dimensiones y los criterios que se elijan. Debe partirse, entonces, de una definición conceptual y operativa, lo cual resulta extraordinariamente difícil (Suñol 2001). Por lo común, la calidad es un reflejo de los valores y metas vigentes en el sistema de atención médica y en la sociedad más amplia de la que ésta es una parte. Donabedian (2001), a partir de una revisión exhaustiva de investigaciones sobre el tema, plantea la importancia del método y el enfoque; hace énfasis en la revisión de historias clínicas y la observación directa de las actividades del médico.

A pesar de las ventajas y la utilidad de las distintas dimensiones en que se basa la evaluación, Donabedian señala como una de las debilidades del método, que en mayoría de los estudios se adopte una definición muy limitada. De manera general se ocupan del tratamiento técnico de la enfermedad y ponen poca atención a los aspectos preventivos y de rehabilitación, así como a la coordinación y continuidad de la atención o el manejo de la relación entre el paciente y el médico; por tanto, plantea en sus conclusiones la necesidad de una exploración conceptual y empírica más amplia. Asimismo, recomienda dejar de preocuparse en exceso por evaluar la calidad para concentrarse en entender el proceso de atención médica propiamente dicho (Donabedian 2001). Una limitación en el 
estudio de este autor es que se circunscribe a la atención otorgada por los médicos, dejando de lado al demás personal de la institución.

La mayoría de los trabajos en este campo se ocupan de medir indicadores que no siempre reflejan la percepción de los usuarios. De acuerdo con Patton (1987, apud Yumi y Magalhães 2007:75), la calidad se hace con matices, con detalles, con la sutileza de las únicas cosas que marcan la diferencia más allá de los puntos en una escala estandarizada. La satisfacción del usuario tiene que ver con sus percepciones como paciente con ciertas expectativas respecto de la institución, de su personal y de las significaciones que le otorga a la experiencia vivida.

Otros estudios más recientes han intentado reflejar las problemáticas de las poblaciones en lo tocante al bienestar y el progreso. Ejemplos de ello son la evaluación de indicadores económicos y materiales en trabajos realizados por la OCDE, el BID, la ONU, la CEPAL y a nivel nacional el INEGI, el Foro Consultivo Científico y Tecnológico, además de distintas universidades públicas y privadas (Hamui et al. 2013). No obstante, los resultados no siempre convergen con la apreciación de los ciudadanos. Algunas investigaciones se han centrado en la población adulta mayor, en quienes características como la edad y el deterioro a causa de la enfermedad son elementos importantes a considerar en la calidad de la atención que reciben.

El tema de la calidad y la satisfacción de los usuarios se ha explorado principalmente desde la utilización de instrumentos cuantitativos, los cuales son limitados para entender la experiencia de quienes hacen uso de los servicios de salud por padecer algún tipo de enfermedad. En este contexto, el acercamiento a estas personas para entender su realidad compleja, tomando en cuenta aspectos multidimensionales, es relevante para disminuir la brecha entre quienes diseñan y ejecutan las políticas públicas y quienes reciben sus beneficios, como es el caso de la salud (Hamui et al. 2013).

En la literatura respectiva se abordan aspectos como la satisfacción de los usuarios en cuanto al trato que reciben del personal de salud, a la infraestructura, a la disponibilidad de los servicios y a los tiempos de espera. De manera general, la calidad está representada por las características del proceso de atención; la mayoría de los usuarios le dan un mayor peso a la relación interpersonal, es decir, ponderan recibir un buen trato; también le dan importancia al contenido de la consulta, pues les interesa tener una buena revisión y un buen diagnóstico. En menor medida es importante la estructura física, humana y la accesibilidad (Ramírez et al. 1998). 
Otros trabajos reportan que la calidad debe ser considerada en dos dimensiones: la técnica y la interpersonal; es decir, no sólo se debe considerar la tecnología médica, sino que ponen énfasis en analizar las interrelaciones entre los usuarios y los prestadores de servicios, así como el contexto espacial donde se desarrolla la atención médica (Campos et al. 2006). Estos estudios privilegian la calidad de la atención en la relación médico-paciente, ya que constituye el sustento de una buena estructura diagnóstica y terapéutica.

Por otra parte, las encuestas de satisfacción de usuarios de servicios de salud representan también un indicador de calidad de la atención sanitaria. Algunas de estas encuestas miden dimensiones referidas a la calidad científica-técnica, la satisfacción de los usuarios y la accesibilidad de los servicios. Aspectos que influyen en una atención deficiente son la falta de personal y de recursos especializados en el caso de la atención a personas mayores. Si bien la mayoría de las investigaciones muestran que alrededor de $80 \%$ de los usuarios están satisfechos con la atención recibida, es necesario tomar en cuenta que el nivel de instrucción y el contexto cultural del usuario juegan un papel determinante en su apreciación de los beneficios y la calidad con que las instituciones de salud otorgan el servicio (Ramírez et al. 1998, Valdez et al. 2005).

En estos mismos estudios se reportan las deficiencias en los servicios que otorga el Seguro Popular, y también las instituciones de seguridad social. Estas deficiencias parecen agudizarse en el caso de la atención a las personas mayores debido al deterioro físico que presentan y que los limita para permanecer largos tiempos de espera en las unidades de salud, o bien, por tener que regresar en

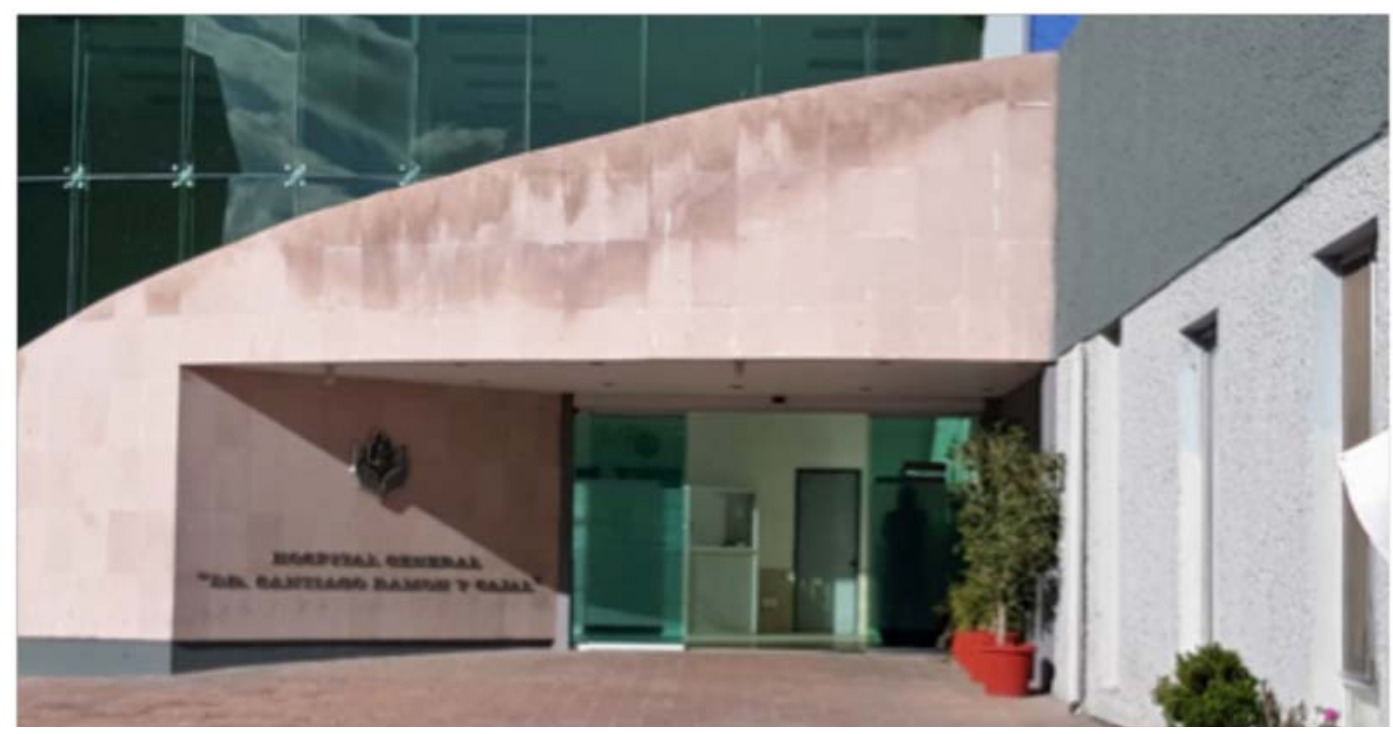


repetidas ocasiones a la institución a causa de fallas en la oportunidad de los servicios y/o de los medicamentos que necesitan. Los estudios con metodologías cualitativas permiten profundizar en las opiniones de los usuarios.

\section{ALGUNAS PRECISIONES TEÓRICO-CONCEPTUALES}

Nos parece importante destacar brevemente en este apartado tres conceptos relacionados en este trabajo: percepciones, experiencias y significados, en el afán de tener mayor claridad en la comprensión de los resultados.

\section{SOBRE LA NOCIÓN \\ DE PERCEPCIÓN}

La teoría de las representaciones sociales aporta elementos de conocimiento para entender la percepción. Los estudios de percepción social se centran en los mecanismos de respuestas sociales y de procesamiento de la información (Araya 2002). El origen de la percepción es meramente sensorial, en tanto la representación social constituye una instancia de propiedades mixtas. Propiedades que pasan de la esfera sensoriomotriz a la esfera cognitiva del objeto percibido a distancia, a una toma de conciencia de sus dimensiones, formas, etc. Representarse una cosa y tener conciencia de ella es todo uno, o casi. A simple vista la percepción es un proceso individual y subjetivo; no obstante, cuando integramos la noción de representación podemos trasladar la percepción al plano social.

La percepción es el proceso que permite al organismo, por medio de los sentidos, recibir, elaborar e interpretar la información proveniente del entorno. El proceso de la percepción es de carácter inferencial y constructivo, generando una representación interna de lo que sucede en el exterior. Con base en la percepción se constituye la experiencia y se formulan opiniones que pueden definirse como el estado de creencia de los individuos y la colectividad respecto de determinado asunto, e implica un juicio de valor (Hamui et al. 2013).

Las percepciones de los actores sociales sobre la calidad de servicios y programas, frecuentemente descuidadas tanto en la organización como en la provisión, se determinan por experiencias inherentes a la vivencia junto con las acciones en cuestión. 


\section{SOBRE LA NOCIÓN DE EXPERIENCIA}

Para los autores del libro Expectativas y experiencias de los usuarios del Sistema de Salud en México. Un estudio de satisfacción con la atención médica (Hamui et al. 2013), la experiencia es una forma de conocimiento o habilidad que se genera por la observación de una vivencia de un acontecimiento o de las cosas que suceden en la vida cotidiana y que conjuga realidad y pensamiento; el sujeto elabora la vivencia desde sus mapas mentales previos, la aprehende, reflexiona, interpreta, transmite y pasa posteriormente a la acción, en la que generalmente se busca bienestar, satisfacción y felicidad.

Desde ese punto de vista, la experiencia de uno es apropiable por otros, «aprender de la experiencia» implica la capacidad de repensar y transmitir la vivencia en un orden de sentido, en estructuras históricas donde los significados se socializan. La experiencia no es un punto de partida o de llegada, es una circunstancia en la que se correlacionan formas de subjetividad, saberes, tradiciones y poderes según las condiciones de posibilidad y el posicionamiento del sujeto.

Larrosa (2003), por su parte, plantea que las experiencias están ligadas al tiempo, a situaciones concretas, a contextos, al cuerpo y a los afectos. A pesar del menosprecio que la racionalidad científica moderna ha hecho de la experiencia por considerarla un tipo de conocimiento inferior, hoy asistimos a su reivindicación en toda la complejidad que conlleva. Es una forma de ser persona y estar en el mundo. La no elaboración de la experiencia es la falta de sentido con relación a la propia vida y la de los demás, y entonces no es posible la transmisión.

Desde el punto de vista de Scott (2001), la experiencia se elabora en forma de relato; si este desaparece, desaparecen los signos y los símbolos significativos, como el lenguaje, que hacen posible el intercambio de experiencias y la cultura. Quedarse sin palabras es perder el sentido y la posibilidad de comprensión de la experiencia, es cuando el sujeto deja de comprender lo que le sucede y rechaza apropiarse y transmitir su experiencia. Se podría pensar la experiencia como la invención, producción y alteración de ámbitos de sensibilidades, de prácticas, de relaciones, de condiciones de enunciación y de discurso, de subjetivaciones, como posibilidad de visibilizar y compartir imágenes dialécticas con otros sin perder la singularidad de los procesos y los acontecimientos al querer generalizar. La singularidad del acontecimiento, donde sucede el procedimiento, al ser puesto en común, lo vuelve una experiencia colectiva. En este sentido, cuando 
los sujetos evalúan cualquier ámbito de la vida o de la experiencia (la familia, la salud, etc.), puede resultar complejo, aunque no imposible en términos objetivos.

\section{SOBRE LA NOCIÓN DE SIGNIFICADO}

El concepto de significado se ha analizado desde distintas ópticas y autores. En este trabajo recuperamos los aportes de Wittgenstein (1988 citado en Hamui et al. 2013), quien plantea que podemos definirlo señalando que el significado de una palabra es su uso en el lenguaje; y agrega que «es importante hacer constar que la palabra significado se usa ilícitamente cuando se designa con ella la cosa que 'corresponde' a la palabra, esto es, confundir el significado del nombre con el portador del nombre». Es la aplicación la que determina el significado, no la imagen mental; y por tanto, intentar averiguar el significado haciendo caso omiso del contexto es una empresa arriesgada, pues cada palabra posee una «familia de significados» irreducible a uno de ellos.

Ahora bien, los significados son subjetivos pues interviene el contexto, la cultura, la personalidad y la historia de vida de los sujetos. No podemos esperar que los significados sobre algo sean homogéneos, siempre habrá diferencias de una persona a otra sobre un mismo asunto. Al respecto de la subjetividad, esta se constituye por un conjunto de representaciones y afectos que funcionan para la producción de sentidos en los que el sujeto orienta su acción, significaciones a partir de las cuales construye su relación con los semejantes y el mundo material que habita, junto a valores e ideales también adquiridos por identificación con el adulto (principalmente el padre, luego los maestros y las figuras sociales de autoridad en una acepción amplia). En este sentido, somos cuerpo, integrado por un cuerpo social y un cuerpo subjetivo en interacción con nosotros mismos y con otros, y aprehendemos y comprendemos el mundo material por medio del lenguaje, de la percepción, de las sensaciones estéticas; es decir, el cuerpo tratado por la medicina no solo como una organización biológica, sino como todo un entramado subjetivo y social. Todos estos elementos constituyen experiencias que ponen en acción la necesidad de la representación y la construcción de un sentido o una significación (Galende, citado en Yumi y Magalhães 2007).

La satisfacción del paciente es fundamental como medida de calidad de la atención, porque proporciona información sobre el éxito del proveedor en alcanzar los valores y expectativas del paciente, que son asuntos sobre los que éste es la autoridad última (Yumi y Magalhães 2007). Por tanto, explorar la satisfacción es 
un elemento valioso para la investigación, para la planificación y transformación de los programas de atención sanitaria de las personas mayores.

Por otra parte, son escasos los estudios que integran la subjetividad de los usuarios de estos servicios. La percepción de los sujetos a este respecto refleja aspectos relacionados con el trato que reciben del personal, con la oportunidad con que se les atiende, con la dotación de medicamentos y tratamientos en tiempo y forma, entre otros. Dejar de contar con estos beneficios restringe a los usuarios de lo más importante para ellos: disminuir el dolor, el malestar y las consecuencias de la enfermedad. Se crea una problemática que merma el bienestar y la calidad de vida de las personas mayores, situaciones que impactan también en su entorno inmediato, en sus relaciones interpersonales.

Los planteamientos mencionados dan pie para formular las siguientes preguntas de investigación:

¿Cuáles son las percepciones de las personas mayores acerca de la atención brindada en las instituciones de salud en la ciudad de Durango?

¿Cuáles son los significados que le atribuyen a la experiencia de acudir a solicitar servicios de salud?

\section{OBJETIVO}

Explorar las percepciones y los significados entre las personas mayores acerca de la atención en las distintas instituciones de salud en la ciudad de Durango.

\section{METODOLOGÍA}

Se trata de un trabajo cualitativo producto de una práctica escolar supervisada con 32 estudiantes de trabajo social de quinto semestre, en que se realizaron 50 entrevistas abiertas a personas mayores (19 hombres y 31 mujeres) que acudieron a recibir atención médica en distintas instituciones de salud en la ciudad de Durango. El contacto con los participantes se realizó en las áreas de consulta externa del Instituto Mexicano del Seguro Social (IMSS), del Instituto de Seguridad Social al Servicio de los Trabajadores del Estado (ISSSTE) y unidades de consulta externa de hospitales y centros de salud de la Secretaría de Salud en donde se cuenta también con el programa del Seguro Popular. 
El tipo de muestreo utilizado fue intencional y se buscó heterogeneidad en cuanto a sexo, edad, tipo de padecimiento y condiciones socioeconómicas. Fueron entrevistados 19 hombres y 31 mujeres $^{5}$ de manera voluntaria y con autorización para ser grabados (se les dio a firmar una hoja de consentimiento informado, aunque algunos participantes no lo consideraron necesario y no se les obligó a hacerlo). El diseño de la guía de entrevista fue realizado entre estudiantes y docentes, integrando las siguientes categorías temáticas: percepciones de satisfacción o insatisfacción con la atención que brindan las instituciones de salud; derivada de esta categoría general se plantearon dos subcategorías que incluían la percepción de satisfacción/insatisfacción acerca del trato que les dan los profesionales de la salud, y acerca de la dotación de medicamentos y servicios; una última categoría se orientó a la opinión sobre los tiempos de espera. De la información de todas las categorías surgen elementos de significado acerca de cómo se interpreta la realidad de las instituciones de salud. Adicionalmente, la guía incluyó algunos datos sociodemográficos como edad, estado civil, personas con quienes vivía la persona mayor, si tenían o no pensión. Previo al inicio del trabajo de campo se realizó una especie de piloteo con personas mayores que habitan viviendas aledañas a la Facultad de Trabajo Social. Las entrevistas tuvieron una duración de entre 40 minutos y una hora 20 minutos; se realizaron principalmente en los hogares de las personas mayores; otros fueron entrevistados en alguna cafetería cercana a las unidades de salud y otros más en algún parque aledaño. Se decidió efectuarlo así en virtud de que la gestión para actuar dentro de las instituciones implicaba esperar un tiempo prolongado que impedía alcanzar las metas del programa escolar; no obstante, el protocolo fue autorizado por la Secretaría Académica y la Coordinación de Investigación de la Facultad. Una vez concluido el semestre fue necesario que las autoras de este trabajo regresaran a practicar una segunda entrevista para ampliar la información de temas que no se profundizaron en la primera.

La duración del trabajo de campo fue de seis semanas, durante abril y mayo de 2014. Un periodo adicional para la segunda visita fue de un poco menos de dos meses. El análisis de los datos se realizó con la asesoría de las investigadoras a partir de la lectura y relectura de los registros, la organización, clasificación y categorización de los relatos. Para ello, se agruparon segmentos de información relacionados con una pregunta o tema de investigación y se etiquetó cada uno

${ }^{5}$ Fue complicado conseguir igual número de hombres que de mujeres, pues de antemano se sabe que quienes más acuden a solicitar consulta médica son mujeres. 
de ellos, para contar así con una lista de códigos. Este procedimiento se realizó en las hojas impresas de los registros de las entrevistas. De esta manera se pasó de una codificación general hasta la construcción de categorías significativas. El ejercicio descrito permitió a los investigadores reconocer y recontextualizar los datos y buscar su interpretación (Coffey y Atkinson 2003:31-63).

Si bien el tiempo dedicado al trabajo de campo no fue tan amplio como lo exige la etnografía clásica, sí fue de utilidad utilizar el enfoque en la medida que permitió observar la conducta no verbal, así como patrones de acción y no acción en los entrevistados, contrastar relatos, así como una descripción sistemática de las características de las variables y categorías de los fenómenos a observar (Martínez 2006). A través del razonamiento inductivo se destacaron elementos comunes y similitudes en las expectativas que depositan en la atención institucional. El enfoque etnográfico no es la panacea, más bien es parte de una tradición respetable de investigación que se está desarrollando con un nivel adecuado de rigurosidad.

\section{RESULTADOS}

Algunos de los participantes, como ya se mencionó en el apartado de la metodología, fueron entrevistados en su hogar y otros en alguna cafetería cercana a la institución de salud. Sus edades fluctuaron entre 59 y 83 años, de los cuales 31 fueron mujeres y 19 hombres; 23 eran casados, 11 divorciados, nueve viudos, seis solteros y solo uno vivía en unión libre. En cuanto a la forma de convivencia, 38 vivían con algún familiar.

Los datos sobre ingreso no fueron respondidos por la mayoría de los participantes; algunos refirieron que sus hijos les han recomendado no dar este tipo de información bajo ninguna circunstancia. ${ }^{6}$ Mostramos a continuación los datos proporcionados.

Como vimos, 12 personas en este grupo viven solas, situación que probablemente se deba a su estado civil, o bien por su voluntad. Las personas casadas tienen mayores oportunidades de contar con la red de apoyo familiar. Por otra parte, predominaron los usuarios del IMSS y del Seguro Popular, por lo que las

\footnotetext{
${ }^{6}$ El problema de inseguridad social que se ha dado en los últimos años limita las respuestas de los participantes sobre algunos temas, tales como datos de la identidad y de los ingresos. Más allá de eso, hubo personas que no aceptaron participar en la entrevista por la desconfianza que prevalece.
} 
opiniones favorables o desfavorables se centran en estas dos instituciones, como vemos en el cuadro 1.

Cuadro 1. Institución de salud donde se atiende y percepción de satisfacción

\begin{tabular}{|l|c|c|c|c|c|}
\hline & IMSS & ISSSTE & SEGURO POPULAR & SSA & OTRO \\
\hline Institución donde se atiende & 21 & 3 & 24 & 2 \\
\hline Percepción de satisfacción & 10 & 1 & 11 & 1 \\
\hline Percepción de insatisfacción & 11 & 2 & 13 & 1 \\
\hline
\end{tabular}

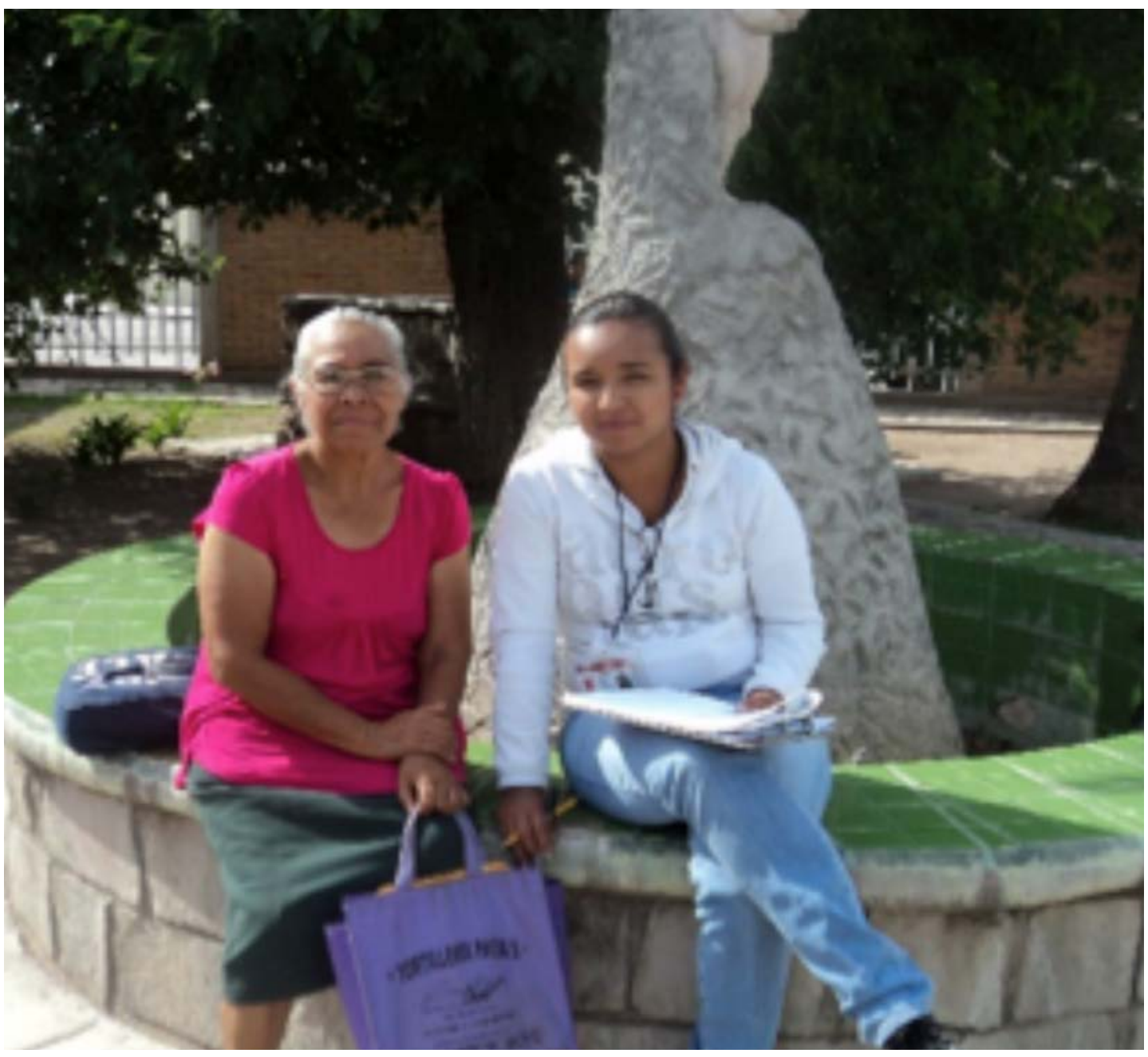




\section{PERCEPCIONES Y SIGNIFICADOS DE SATISFACCIÓN/INSATISFACCIÓN POR LA ATENCIÓN RECIBIDA}

Las personas entrevistadas mostraron opiniones tanto positivas como negativas con respecto a la atención que reciben en las instituciones de salud a las que acuden. Las opiniones positivas representaron casi la mitad de las entrevistas realizadas y en la otra mitad resultaron tener más peso las opiniones negativas, resultados un tanto diferentes a lo que se reporta en la mayoría de los estudios sobre satisfacción y calidad de la atención en los servicios de salud, donde se identifica $80 \%$ en promedio de opiniones positivas (Peña et al. 2009). Las percepciones de satisfacción/insatisfacción se agruparon en dos grandes categorías:

- Respecto al trato del personal, especialmente en la relación médico-paciente.

- Respecto a la disponibilidad tanto de servicios como de medicamentos.

Una tercera categoría que incluye a las dos anteriores la definimos con los significados que atribuyen los participantes a las experiencias mencionadas. En un primer momento mostramos las percepciones de satisfacción.

\section{SATISFACCIÓN POR EL TRATO DEL PERSONAL}

En este renglón de atención y trato se exploraron las prácticas y actitudes del personal, especialmente del trato de los médicos. Los relatos que seleccionamos fueron los siguientes:

Muy bien me atendieron ahí (Hospital General) los doctores, y el doctor el que me atendió el que me hizo la cirugía, que no era muy amable que digamos, pero me atendió en el aspecto de que me hizo la cirugía bien, aunque a veces si se portaba medio mal, pero sí me atendía (Consuelo, 60 años).

Atienden primero a los de la tercera edad (en el centro de salud 1), porque dicen, primero van a pasar los de la tercera edad y entonces ya se queda la demás gente que está esperando enseguida de los de la tercera edad, entonces sí me ha gustado, pero como le digo, todavía hasta ahorita vamos bien, ahorita por lo pronto sí estoy bien (Epifanía, 70 años. Con derecho a IMSS y a Seguro Popular).

No, pues muy buena, es muy buena (la atención en el Seguro Popular) como le decía yo traía problema de los ojos y gracias a dios ya me hicieron una operación ¿verdad?, que, si no ha sido por ellos pues acá por otro lado no, no lo hubiera podido obtener o realizar, pues a veces sale caro (Gilberto, 64 años). 
De manera predominante, los entrevistados eran usuarios del Seguro Popular, $y$, en segundo lugar, del IMSS; como consecuencia, tanto las opiniones positivas como las negativas fueron más frecuentes hacia las unidades del Seguro Popular y del IMSS que para los que se atienden en el ISSSTE o la Secretaría de Salud. Lo que destaca el primer relato es que la gente valora más resolver su problema de salud por encima del buen trato, es decir, aunque no haya recibido un trato cálido y amable, se reconoce que hubo buen resultado en la práctica médica.

\section{SATISFACCIÓN RESPECTO DE LA DOTACIÓN DE MEDICAMENTOS Y SERVICIOS}

La atención médica es completa cuando se accede a los tratamientos que coadyuvan a mejorar la salud. A lo que se hace mayor referencia en las entrevistas es a la dotación oportuna de medicamentos, como vemos en los ejemplos:

Por suerte a mí no me ha faltado mi medicamento, yo siempre que voy me lo entregan completo, yo he visto que a otras personas las mandan a otro lado a conseguir su medicamento y a mí no, a mí no me ha tocado eso todavía (Juana, 67 años).

He tenido la buena suerte de recibir atención en el Seguro Social, que he estado internado un par de veces y este... mi visita la acabo de hacer ahora... la última, el día 4 de este mes, con muchas atenciones (Jorge, 85 años).

Yo recibí muy buen servicio en el hospital (de la Secretaría de Salud), yo he oído, he oído así en las televisiones que, que pues es un pésimo servicio, yo para mí no, a mí me atendieron muy bien por todos lados (Jesús, 70 años).

En estos relatos llaman la atención dos aspectos: uno, que en buena medida las personas entrevistadas que dieron una opinión satisfactoria de la atención recibida atribuyen a la suerte el hecho de haber recibido un buen trato y todos los medicamentos. Esta perspectiva deja de manifiesto que una buena atención de salud no es algo que se establezca por regla, por norma, sino más bien por probabilidad. Los estudios revisados mencionan que aproximadamente $80 \%$ se encuentran satisfechos con la institución. En este trabajo, por su naturaleza cualitativa, no podemos comparar los datos; sin embargo, resulta interesante que alrededor de la mitad de los participantes opinen diferente, lo cual nos hace suponer que ellos hablan desde su experiencia personal, que sus respuestas se construyen con base en su condición social, en su contexto cultural, en su per- 
cepción como personas con derechos, o, por lo contrario, como personas a las que se les otorga un beneficio social que deben agradecer.

Los participantes expresan que las percepciones positivas del trato y la atención que reciben no sucede con todos los usuarios, están conscientes de que una parte de la población no opina igual. Por ello la insistencia en denominar su experiencia como «buena suerte», además del hecho de contar con un «conocido» en las instituciones: "Pues yo creo que hay preferencias... a veces llegan [personas] 'conocidas' de las asistentes, y uno tiene su lugar y llega alguna 'conocida' y la pasan... y uno sigue esperando». Acceder a una atención rápida y de calidad por ser «recomendados» de algún empleado de la institución implica un obstáculo para que otros usuarios obtengan la atención con la oportunidad a la que tienen derecho. Esto permite reflexionar que sobre los tiempos de espera los participantes no expresaron opiniones positivas, lo cual hace suponer que ese es un aspecto complejo de resolver para los responsables de las instituciones de salud. A continuación, mostramos algunos resultados.

\section{INSATISFACCIÓN POR LA ATENCIÓN RECIBIDA}

Las personas entrevistadas opinan sobre la atención que se les presta en las instituciones de salud. Las respuestas se clasificaron en función de las categorías establecidas para este trabajo. En primera instancia mostramos opiniones de insatisfacción por la atención y el trato que reciben en la institución, y en un segundo momento, inconformidades respecto de los tiempos de espera y la escasez de medicamentos.

\section{LA ATENCIÓN Y EL TRATO}

Uno de los aspectos más importantes que generan insatisfacción en los usuarios es la ausencia de trato adecuado por parte del personal de salud, específicamente de los médicos. Las personas mayores consideran importante expresar a su tiempo y a su ritmo las dolencias que los aquejan, sus dudas y sus expectativas acerca de los tratamientos. Sus condiciones de existencia requieren una atención cálida, paciente, que refleje aceptación y que les brinde una esperanza realista de mejoría. No todo se limita a obtener una consulta, o una receta, se espera 


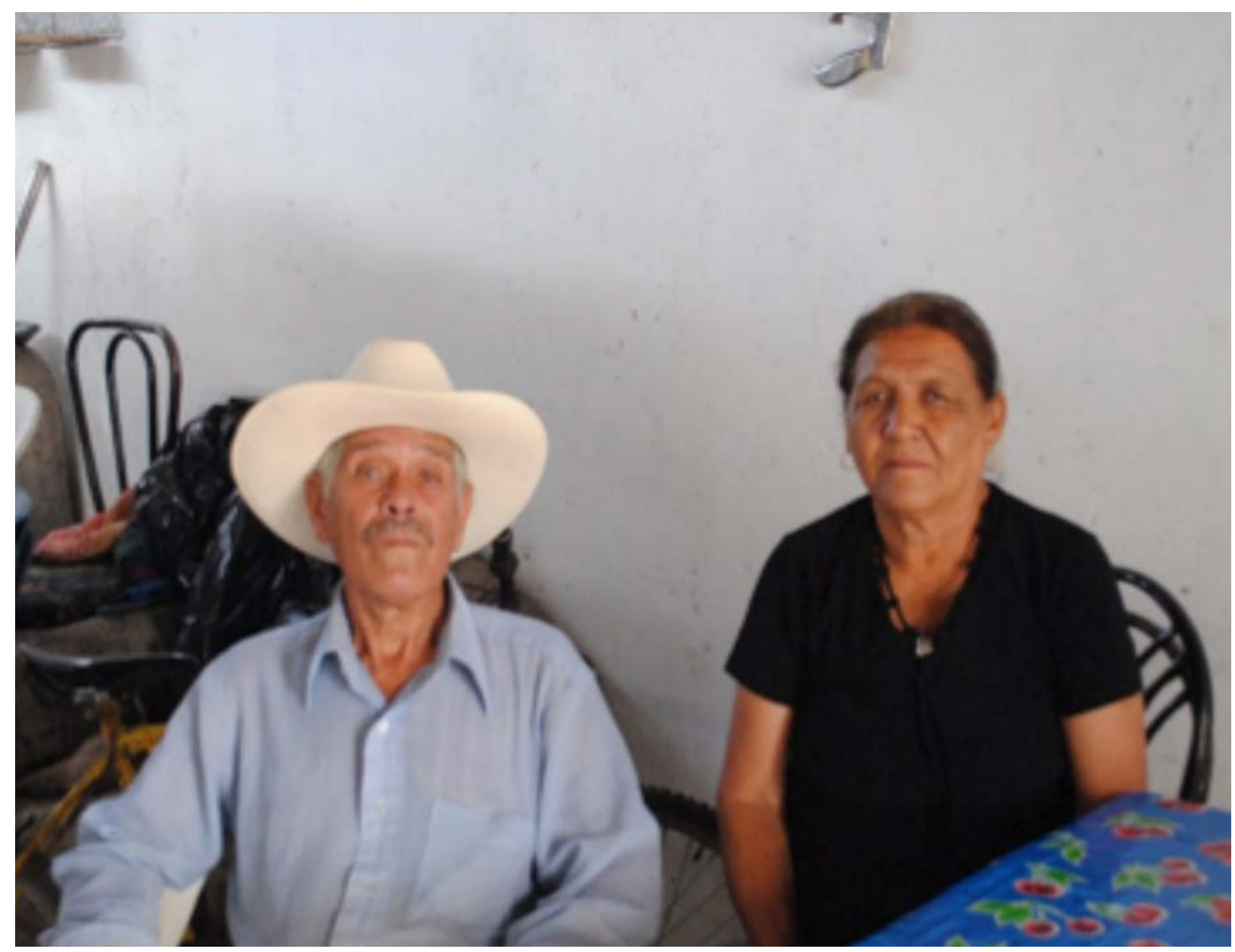

también encontrar una actitud comprensiva frente a las dolencias y motivación para continuar con la lucha por la salud, por lo que quede de ella.

¿El doctor? No, no, él hace su consulta muy rápido y ya... o sea no, está escribe y escribe y él no, pues él ya casi las citas salen en automático, son las mismas cosas si uno lleva otra complicación pues no, es lo mismo, el mismo medicamento, no le cambia, no le cambia (Susana, 65 años).

No, no, una doctora que le toca a mi mamá hasta sé el nombre, pero no lo digo... ande, no te voltea ni a ver nomas agarraba el papel y 'aquí tiene, si viene por su medicina, tenga' (Dora, 66 años).

En la relación médico-paciente es importante la forma de ser escuchados, la mirada, la actitud de aceptación y comprensión, cómo se conduce el profesional para proporcionar al paciente la información que éste requiere, cómo le aclara 
dudas y lo motiva a seguir con el tratamiento; de esta manera la adherencia al tratamiento será más efectiva. Se requiere entonces una mayor capacitación a los profesionales de la medicina orientada a mejorar el trato a las personas mayores que los necesitan (Gutiérrez y Lezana 2013).

Pues mire, yo respeto mucho la labor del doctor y aparte de eso pues yo sé que pues ellos, ellos pues a lo mejor en ese instante, pues ellos a lo mejor quisieran dedicarle más tiempo a uno, pero ellos también están bajo presión que de cierto tiempo a cierto tiempo tiene que pasar el paciente, entonces ellos también se restringen a lo mejor a, a examinarlo bien a uno [...] Pues si se me hace poco el tiempo, que nos deberían de dar un poquito más de tiempo para contarle al doctor pues con más exactitud nuestros problemas que llevamos (Socorro, 67 años).

Los usuarios tienen una percepción de los problemas estructurales que enfrentan las instituciones de salud y tratan de justificar las deficiencias que observan. Probablemente escuchan los discursos del personal y tratan de entender la presencia de otros factores que impiden a los médicos brindarles el tiempo suficiente (situación muy común en el IMSS).

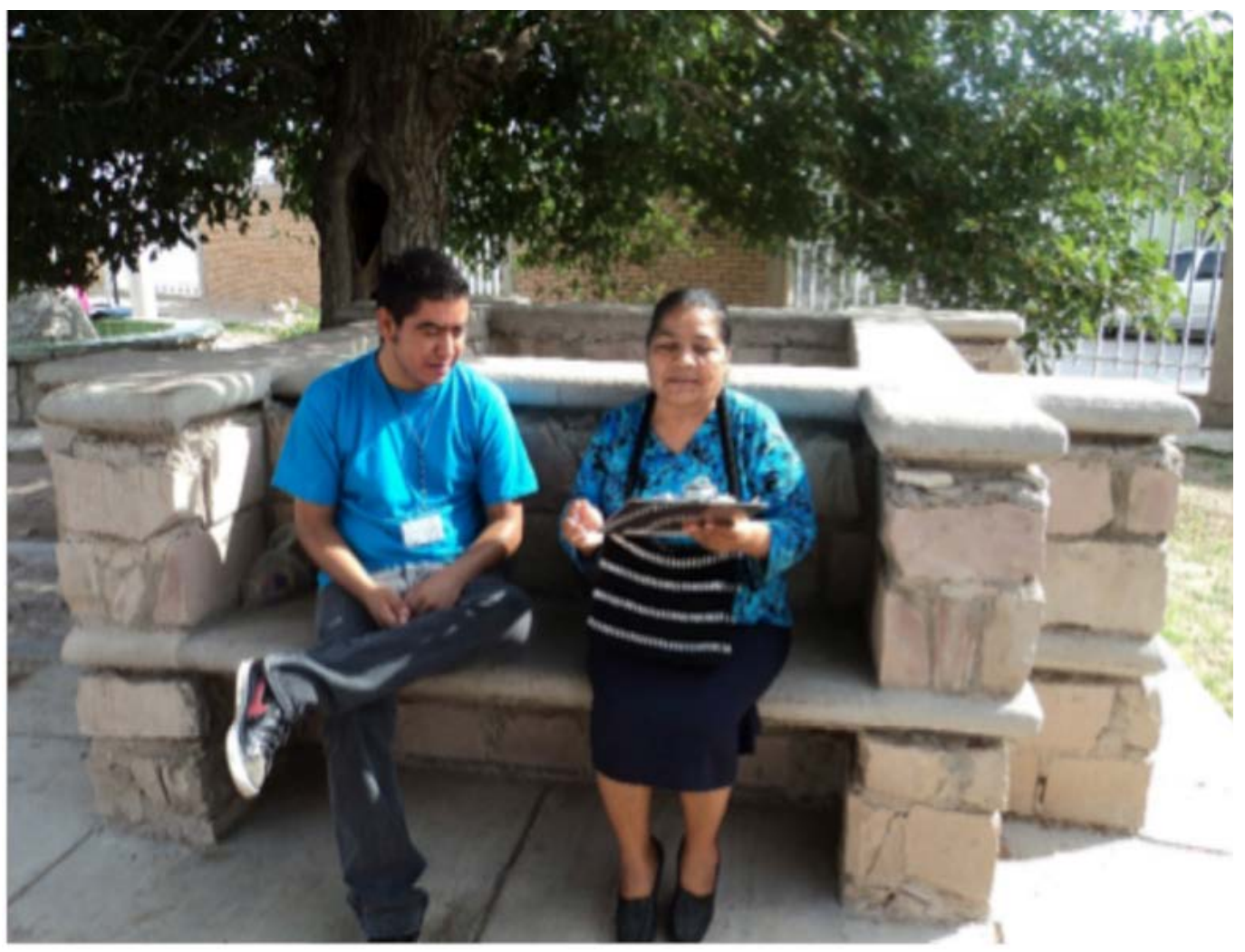


Pues con las enfermeras, hay de todo, unas 'gestosas', unas amables, unas lo tratan a uno con cariño, otras 'secotas, bien feas' ... pues que contraten a personal que de veras traten bien a las personas, porque, aunque uno ya es mayor no deja de ser persona (María del Rosario, 62 años).

Pues la verdad 'hay que tener suerte' para tener una buena atención, porque si le toca la de malas pues le toca las burocracias y pos recibe uno malas atenciones y hay veces que hasta regaños y malos modos de las personas que lo atienden a uno (Rafael, 66 años).

Nuevamente el elemento "suerte» aparece cuando de forma general este entrevistado habla de la mala atención recibida. A pesar de que los participantes identifican problemas de burocracia y mala calidad en la atención no logran profundizar en las deficiencias, y en ese sentido no se emprenden acciones encaminadas a exigir sus derechos.

\section{LOS TIEMPOS DE ESPERA}

Los tiempos de espera constituyen uno de los aspectos más importantes que los participantes incluyen en la opinión positiva o negativa del trato y la atención institucional. Independientemente de que para nadie es agradable invertir largos periodos de tiempo para recibir atención de cualquier índole, las personas mayores, dada su condición de fragilidad, lo resienten más. Existen ciertos padecimientos como la artritis reumatoide, los problemas de columna o el vértigo, entre otros, que limitan a las personas a permanecer sentadas o de pie por mucho tiempo, lo cual incrementa el malestar por la enfermedad. De igual forma, el tiempo de espera para que se realicen estudios o acudir con especialistas es motivo de insatisfacción en los participantes. A continuación, presentamos los siguientes testimonios:

Pues, lo único que pues si más o menos [se tarda] es sacar la ficha, esperar para el día que le dan a uno, si no hay para ese día, yo me sentía molesta porque tenía que irme a las 6:00 am para sacar la ficha y tenía que regresar a las 2 o 3 de la tarde para la consulta (Rita, 76 años).

Cuando uno viene, uno quisiera que lo atendieran rápido, mire, porque para sacar citas hace uno una 'colona' [larga fila], ahí se está uno una hora formado para sacar 
cita, y luego lo mandan a uno a sacar su medicamento y otra fila, y... pues a lo mejor si hubiera más personal pues era mejor, ¿no? (Juana, 67 años).

Tengo que irme desde las siete de la mañana para dejar la tarjeta...y a las dos, no, me voy a la una. A la una me voy otra vez para estar ahí a las dos que llega la doctora, entonces llega la doctora y la muchacha enfermera que checa la presión esa, ipues todavía no llega! Y a veces así lo meten a uno sin checar la presión ni la azúcar. ¡Como para checar la azúcar nunca tienen con qué!, yo tengo mucho que no me la checan la azúcar porque no tienen con qué checarla (Socorro, 65 años).

En este último relato se identifican dos tipos de insatisfacción, una tiene que ver con el tiempo que los usuarios invierten en la solicitud de la consulta y la otra con las fallas del personal para la revisión preliminar. El dato se relaciona con lo que evalúan ciertos indicadores de CONEVAL respecto de los servicios de salud en nuestro país, donde se reporta que, en 2012, a $46.9 \%$ de las personas de 30 años y más en los últimos 12 meses no se les habían tomado muestras de sangre para medir niveles de glucosa. En el mismo caso, pero con 30.7\%, a ese mismo grupo no se le midió la presión arterial (CONEVAL 2013).

\section{FALTA DE OPORTUNIDAD EN ENTREGA DE MEDICAMENTOS Y OTROS SERVICIOS}

Distintos estudios nacionales sobre calidad y satisfacción de la atención en salud (Juárez et al. 2014, Ramírez et al. 1998), declaran que uno de los puntos que más influyen en la satisfacción/insatisfacción de los usuarios es la falta de disponibilidad de medicamentos, seguido de algunos servicios que no son cubiertos.

No hay mi medicamento en la farmacia y me traen con vueltas y vueltas ahorita, no es justo que uno, que, pues está discapacitado, como le digo, señorita, y este, pues, para comprar el medicamento pues no se puede (Amalia, 62 años).

No, si dondequiera es lo mismo, es lo mismo, ahorita se está batallando en el IMSS, en el ISSSTE, en el DIF, ahorita pos' también hasta las personas que tienen su seguro popular, digo, ¿para qué dan seguro popular si el seguro popular no cubre muchos medicamentos, una radiografía, a veces no la cubre, pues si a veces hasta una operación no la viene cubriendo (Amalia, 62 años). Esta misma entrevistada agrega lo siguiente:

Yo batallé con el médico que me iba a operar porque acudía yo y este y pos', el doctor no iba, que 'ora' pidió el día... y que no vino a trabajar... y que esto y que lo otro... 
y pues ahí se la lleva, le digo hasta que al fin este ya... encontré al doctor y pues ya, medio así, medio molesto, pues ya me dio la cita, pues véngase tal día para operarla.

No, en el Seguro casi no me gustó... en el Seguro (IMSS), pero por eso, porque iba yo muy mala y no me atendieron (Epifania, 70 años).

En estos dos últimos relatos se enfatiza que además de no recibir el servicio, a ello se agrega el ausentismo y el trato inadecuado del personal médico de base (sobre todo en fin de semana). La atención entonces es otorgada por los médicos en formación (residentes o internos) sin la supervisión de los titulares, lo que conlleva algunos riesgos que ponen en peligro la salud de los usuarios, pues si bien aquellos tienen la capacidad de atender los problemas de los pacientes, también es cierto que hay procedimientos médicos que requieren la experiencia de los médicos de base.

Bueno pos que pusieran gente capacitada porque inclusive tienen allí muchos que salen de la escuela y son aprendices, allí se enseñan con la gente, echan a perder mucha gente, sí, y, este, se necesita gente más, pos' sí, que esté más estudiada en la medicina (Arturo, 69 años).

Pues sí, mire, una vez que fui, iba grave pero igrave de la vesícula! Bastante mal, me dijo, llegué a urgencias en el ISSSTE y luego ya me examinó el doctor y me dice 'pues viene muy mal, señora, su vesícula está bastante inflamada, está para reventarse, pero pues es sábado y no hay médico que la pueda atender' ... yo sentía que ya no podía soportar aquel dolor tan fuerte y, la verdad, yo me salí muy molesta (Rita, 76 años).

Otro caso relacionado es el de la señora Olga, de 73 años, con atención en el Seguro Popular. Ella estuvo a punto de abandonar el hospital a causa de negligencia en el procedimiento que necesitaba:

Me tocó la mala suerte de que me puse mal de la vesícula en fin de semana, ya estaba programada, pero me puse mala antes, y era sábado, y todo el fin de semana con suero, y el analgésico ya no me hacía efecto... que porque no había quirófano, y que no estaba el especialista... me atendieron puros estudiantes... no daban una... el domingo en la tarde ya no aguantaba y les dije: 'me voy... denme la hoja para firmar el alta voluntaria y mi hija los amenazó con reportarlos, y de rato llegan y dicen: ya está listo el quirófano y me meten, y mire cómo me dejaron... me tuvieron que volver a abrir, porque se me infectó... la que me operó no era especialista, era de esas que están aprendiendo... desde entonces no he estado bien, he tenido que ir con el médico particular. 
Estas experiencias reflejan que no por estar afiliado al Seguro Popular se tiene la certeza de atenderse en las instalaciones de la Secretaría de Salud. Un buen número de afiliados busca atenderse en clínicas privadas (CONEVAL 2014). Es decir, al no recibir la atención que necesitan, o bien, al no obtener los resultados esperados, los usuarios recurren a la medicina privada con los consecuentes gastos que merman la economía de las familias.

\section{DISCUSIÓN}

Múltiples reflexiones surgen alrededor de los relatos de los participantes. Un primer aspecto a destacar es que aproximadamente la mitad de ellos se perciben insatisfechos con la atención que reciben. Este dato resulta interesante pues si bien no se puede comparar con resultados de estudios cuantitativos (donde se identifican porcentajes mayores de satisfacción), es conveniente considerar lo que se expresa en este estudio. También habría que reflexionar acerca de si estas problemáticas ocurren con la población en general o si se agudiza con las personas mayores.

Respecto a las percepciones de satisfacción en el trato, la atención y el suministro oportuno de medicamentos y otros servicios, debemos considerar que hay experiencias reales de buena atención en muchos de los usuarios de los servicios sanitarios. Lo interesante aquí es valorar la percepción de aquellos que atribuyen a la suerte el hecho de ser bien atendidos. Es decir, no le dan un significado relacionado con sentirse personas con derecho a recibir una atención adecuada. La imagen de sí mismos de ciudadanos con derechos parece no estar presente.

Por otro lado, los aspectos que generan insatisfacción en los usuarios son los mismos que distintos estudios destacan como esenciales para lograr una percepción positiva de la atención sanitaria: principalmente el buen trato por parte del médico, un buen diagnóstico y un tratamiento que alivie los dolores. De ahí que si sucede lo contrario vendrá la insatisfacción, el enojo y la frustración. Como ya se mencionó anteriormente, es importante valorar la percepción subjetiva de los usuarios, pues ellos llevan ciertas expectativas respecto de la institución y de los profesionales que los atienden, por lo tanto, esperan tener una experiencia positiva que debiera ser cumplida. Como sugiere Donabedian (2001), es mejor poner atención a los procesos de atención que a los procesos de evaluación. 
Las opiniones de insatisfacción de los participantes expresan molestia cuando no se sienten escuchados o comprendidos por los médicos, cuando no les dedican el tiempo suficiente o no les dan una explicación clara de su padecimiento o un tratamiento efectivo. Habría que reflexionar sobre las condiciones en que trabajan los médicos y el personal de la institución en cuanto al número de pacientes que se les asigna, en cuanto a la disponibilidad de las citas y los servicios. Los usuarios identifican fallas estructurales como es la gran cantidad de pacientes que los médicos tienen que atender, pero también ubican la actitud inadecuada del personal. Por otra parte, la falta de disponibilidad de personal en fines de semana, de medicamentos y de algunos servicios viene a agudizar la atención y el seguimiento adecuado de la enfermedad, y eso repercute en la calidad de vida de los usuarios. A ello se agregan los largos tiempos de espera para recibir la atención médica.

Cabe destacar la actitud de las personas mayores en tanto no se identifica una respuesta más activa ante las fallas, es decir, no expresan una queja de manera formal, una exigencia ante las autoridades, más bien se aprecia una especie de aceptación, una actitud conformista o de «mala suerte», y la forma en que muchos lo resuelven es acudir a la medicina privada, con el consiguiente deterioro de su economía.

Si bien en este trabajo se han mostrado resultados que reflejan satisfacción con la atención recibida en las instituciones de salud, es importante repensar en los resultados de insatisfacción, pues estos no deberían presentarse en la proporción en que aparecen en este y en otros estudios. Se debe considerar cómo mejorar los aspectos estructurales de las instituciones de salud. El recurso humano, técnico y material es insuficiente y poco capacitado ante la fuerte demanda de atención de personas de la tercera edad. De manera general, se pide a los médicos atender 20 o más pacientes en unas cuantas horas con el fin de responder a la demanda en cantidad, a costa de la calidad de la atención y de las necesidades de los usuarios, en este caso, personas mayores. En cuanto al Seguro Popular, el problema es el incremento en el número de afiliaciones y seguir atendiendo casi con el mismo recurso humano, de equipo y de infraestructura; por lo tanto, la disponibilidad y la calidad decaen en detrimento de la salud de las personas en general y de los adultos mayores en particular.

Otro elemento de análisis es el que se refiere a la actitud de los profesionales de la salud, en los aspectos éticos y de humanismo. Coincidimos con Kalache (2014, citado en Rivero 2014:178): cuando plantea interrogantes como: ¿Que 
están aprendiendo los estudiantes de medicina? ¿Cómo se están formando? Porque es cierto que en los hospitales existe rechazo y también iatrogenia (alteración, especialmente negativa, del estado del paciente, producida por el médico); no podemos negar comentarios del tipo: «Ay, qué mañana tan pesada, es el séptimo anciano que estoy viendo hoy». Se tiene una gran ignorancia en el manejo de las personas adultas mayores y se les está afectando, a veces arriesgando su vida por ignorancia. Los profesionales de las instituciones de salud deben evitar decir a los pacientes «pero a su edad, ¿qué se puede esperar?» (Ruelas, apud Rivero 2014). Las personas senescentes traen consigo un riesgo alto que es reflejo de riesgos acumulados a lo largo de toda la vida. Por otra parte, muchos de los padecimientos que se atienden en los hospitales y los costos que estos generan podrían evitarse con una atención primaria más efectiva, pero como menciona Ruelas (apud Rivero 2014), pareciera que los sistemas de salud han sido diseñados para atender personas enfermas en lugar de adoptar un enfoque hacia el cuidado del envejecimiento, hacia el cuidado de la salud desde etapas previas a la vejez.

\section{CONCLUSIONES}

Las percepciones de insatisfacción de los usuarios deben poner un foco de alerta a los responsables de las instituciones sanitarias. Tanto las fallas de tipo estructural como las actitudes profesionales inadecuadas se conjugan para generar esa percepción en los pacientes. Es un hecho que no se han cumplido los planteamientos realizados en los diversos pactos internacionales y las propuestas de expertos en materia de salud para personas mayores. ' La calidad de la atención de la salud de este sector de la población aún no alcanza sus mejores estándares. Es necesario un modelo diferenciado que tome en cuenta que hay personas mayores con mejores condiciones de salud y otras en condiciones crónicas de

\footnotetext{
7 Para ejemplificar, retomamos los acuerdos y la legislación en materia de atención a las salud del Plan de Madrid (OMS 2005); en Latinoamérica, la declaración de Brasilia (CEPAL 2011) y la Carta de San José (CEPAL 2012), además de la Ley de Protección de los Derechos de las Personas Adultas Mayores en México y dos de los principios básicos que aparecen en el documento: «Envejecimiento y salud: una propuesta para un plan de acción» donde se plantea: 1) «más que la búsqueda de la plena curación, reconocemos la calidad de vida como el objetivo fundamental. Desde esta visión, recuperamos la tradición hipocrática donde lo importante no es solo curar sino también cuidar y confortar» y, 2) «es necesario contar con programas diferenciados para los distintos grupos funcionales de adultos mayores: tanto para las personas mayores sanas, como para aquellas frágiles y para quienes están enfermos o son dependientes (Gutiérrez y Lezama 2013).
} 
enfermedad que requieren una atención distinta y con mejores estándares de calidad (Ruelas, apud Rivero 2014).

Se entiende que la salud es un derecho, que debe ser universal. No obstante, la concepción de «derecho» pareciera estar ausente del pensar y del actuar de los usuarios y de los ejecutores de la política sanitaria. México requiere políticas públicas de prevención y cuidado de la salud enfocadas a los adultos mayores, como parte de una estrategia integral. Como plantea Kalache citado por Ruelas: conviene fomentar el autocuidado, el cuidado familiar, de los amigos y vecinos, la atención primaria y al final el cuidado institucional. Una cultura del envejecimiento debe estar orientada al cuidado, más allá de la prevención; hay que incluir el reconocimiento a la cuidadora e incentivar al hombre a participar más en esa labor (Ruelas, apud Rivero 2014).

El cuidado institucional compete al Estado, que tiene la responsabilidad social de velar por la salud de los ciudadanos; es decir, el cuidado en su dimensión social más amplia. Pero, además, un gran número de personas adultas mayores (aquellas que entran en condiciones de fragilidad) tienen una necesidad subjetiva de ser cuidados, de ponerse en manos del "otro» al que configuran como «cuidador» de su salud, de su integridad física y emocional y de su vida, en última instancia. Como plantean Yumi y Magalhães (2007): explorar la satisfacción constituye un elemento valioso para la investigación, para la planificación y transformación de los programas de atención de salud, en este caso de personas mayores. Finalmente, la propuesta es envejecer con salud, porque envejecer sin salud es un costo que no nos merecemos.

\section{FUENTES DE CONSULTA}

Albalá, Cecilia, María Lucía Lebrao, Esther María León, Roberto Ham-Chande, Anselm J. Hennis, Alberto Palloni, Martha Peláez y Omar Pratts, 2005, «Encuesta Salud, Bienestar y Envejecimiento (SABE): metodología de la encuesta y perfil de la población estudiada», Revista Panamericana de Salud Pública, 17(5/6), pp. 307-322.

Araya, Sandra, 2002, «Las representaciones sociales: ejes teóricos para su discusión», Cuadernos de Ciencias Sociales, San José, FLACSO.

Campos Navarro, Roberto, Afganis Juárez Elia Nora, y Daniel Torrez, 2006, "Calidad de los servicios de medicina familiar según adultos mayores del sureste de la ciudad de México», Archivos en Medicina Familiar, 8(2), pp. 131-136, <www.redalyc.org/ articulo.oa?id=50780210> [consulta: el 12 de agosto de 2008]. 
Casen, 2013, «Encuesta de Caracterización Socioeconómica Nacional», Observatorio Social, Ministerio de Desarrollo Social, Gobierno de Chile, http://observatorio.ministeriodesarrollosocial.gob.cl/index.php [consulta el 29 de marzo de 2016].

Coffey, Amanda y Paul Atkinson, 2003, Encontrar el sentido a los datos cualitativos, Colombia, Universidad de Antioquia.

Comisión Económica para América Latina y el Caribe, 2012, "Carta de San José sobre los derechos de las personas mayores de América Latina y el Caribe», Conferencia regional intergubernamental sobre envejecimiento en América Latina y el Caribe, $<$ www.cepal.org/celade/noticias/paginas/1/44901/CR_Carta:ESP.pdf> [consulta: 21 de marzo 2014].

Comisión Económica para América Latina y el Caribe, 2011, "Declaración de Brasilia». Segunda Conferencia Regional Intergubernamental sobre Envejecimiento en América Latina y el Caribe: hacia una sociedad para todas las edades y de protección social basada en derechos, pp. 1-10, <www.cepal.org/publicaciones/xml/0/32460/ lcg2359_e.pdf > [consulta: 13 de abril de 2013].

Consejo Nacional de Evaluación de la Política de Desarrollo Social, 2013, Informe de Pobreza en México 2012, México, CONEVAL.

Indicadores de acceso y uso efectivo de los servicios de salud de afiliados al Seguro Popular, México, CONEVAL.

Donabedian, Avedis, 2001, «Evaluación de la calidad de la atención médica», Revista Calidad Asistencial, suplemento 16(1) pp. 11-27, <www.fadq.org/wp-content/ uploads/2016/02/Monografico-Avedis-1parte.pdf> [consulta: 12 de febrero de 2016].

Encuesta Nacional de Salud y Nutrición (ENSANUT), 2012, Resultados Nacionales 2012, Cuernavaca, Instituto Nacional de Salud Pública, <www.insp.mx/ensanut/resultados_ensanut.pdf> [consulta: 15 de mayo de 2013].

Gutiérrez, Luis Miguel y David Kershenobich, 2012, Envejecimiento y salud: una propuesta para un plan de acción, México, Academia Nacional de Medicina-INGER-UNAM.

Gutiérrez, Luis Miguel y Lezana Miguel Ángel, 2013, «Propuesta para un plan de acción en envejecimiento y salud», México, INGER (Serie de Cuadernillos de Salud Pública).

Hamui, Liz, Ruth Fuentes, Rebeca Aguirre y Omar Ramírez, 2013, Expectativas y experiencias de los usuarios del sistema de salud en México. Un estudio de satisfacción con la atención médica, México, UNAM.

Instituto Nacional de Estadística y Geografía, 2013, Mujeres y Hombres en México 2013, México, INEGI.

Juárez, Clara, Margarita Márquez, Nelly Salgado, Blanca Estela Pelcastre, María Guadalupe Ruelas y Hortensia Reyes, 2014, «La desigualdad en salud de grupos vulnerables de México: adultos mayores, indígenas y migrantes», Revista Panamericana de Salud Pública, 35(4), pp. 284-90.

Larrosa, Jorge, 2003, "La experiencia y sus lenguajes», conferencia dictada en Serie de Encuentros y Seminarios, Barcelona, Departamento de Teoría e Historia de la Educación, Universidad de Barcelona, <www.me.gov.ar/curriform/publica/ oei20031128/ponencia_larrosa.pdf> [consulta: el 15 de junio de 2006]. 
López Muñoz, Eunice y Nora M. Torres Carrillo (coords.), 2012, Aspectos moleculares del envejecimiento, México, INGER-Secretaría de Salud.

Martínez, Miguel 2006, Ciencia y arte en la metodología cualitativa, México, Trillas.

Muñoz, Onofre, Carmen García y Luis Durán, 2004, La salud del adulto mayor, México, Instituto Mexicano del Seguro Social.

Organización Mundial de la Salud, 2005, «Plan de Acción Internacional sobre el Envejecimiento: Informe sobre su ejecución». 58 Asamblea mundial de la Salud, pp. 1-6, <apps.who.int/gb/archive/pdf_files/WHA58/A58_19-sp.pdfhttp://apps.who.int/ gb/archive/pdf_files/WHA58/A58_19-sp.pdf> [consulta: el 15 de junio de 2006].

Peña, Belem, Margarita Terán, Fernando Moreno y Manuel Bazán, 2009, «Autopercepción de la calidad de vida del adulto mayor en la Clínica de Medicina Familiar Oriente del ISSSTE», Revista de Especialidades Médico-Quirúrgicas, 14(2) pp. 53-61, <redalyc. uaemex.mx/src/inicio/ArtPdfRed.jsp?iCve=47312308002> [consulta: 21 de febrero de 2014].

Promoción de la Salud y Determinantes Sociales 2013-2018, Programa Sectorial de Salud, México, Secretaría de Salud <www.promocion.salud.gob.mx/dgps/descargas1/programas/Promocion_de_la_Salud_y_Determinantes_Sociales.pdf $>$ [consulta 12 de marzo de 2016].

Ramírez-Sánchez, Teresita de Jesús, Patricia Nájera-Aguilar, Gustavo Nigenda-López, 1998, «Percepción de la calidad de la atención de los servicios de salud en México: perspectiva de los usuarios», Salud Pública de México, 40(1), pp. 3-12.

Rivero, Octavio, 2014, Salud y envejecimiento. Situación actual, retos y propuestas, México, UNAM.

Román Morales, Luis Ignacio y Enrique Valencia Lomelí, 2012, «Pobreza, desigualdad de oportunidades y políticas públicas en México: el combate contra la pobreza y la desigualdad», en Perticara, Marcela y María Lucia Rodríguez, (coeds.), Pobreza, desigualdad de oportunidades y políticas públicas en América Latina, Brasil, KAS-SOPLA, pp. 109-123.

Rubio, Gloria y Francisco Garfias, 2010, «Análisis comparativo sobre los programas para adultos mayores en México», en Series de la CEPAL, Santiago de Chile, CEPAL, <repositorio.cepal.org/bitstream/handle/11362/6166/S1000316_es.pdf?sequence=1> [consulta: 5 de abril de 2012].

Ruelas, Ma. Guadalupe y Nelly Salgado, 2008, «Lesiones accidentales en adultos mayores: un reto para los sistemas de salud» Salud Pública de México, 50(6), pp. 463-471, <scielo.org.mx/scielo.php?script=sci_arttext\&pid=S0036> [consulta: 13 de marzo de 2016].

Salgado de Snyder, Nelly, 2007, "Los retos del envejecimiento y la pobreza en México para la protección social en salud» Salud Pública de México, vol. 47, pp. 345, <www. redalyc.org/articulo.oa?id=10649135> [consulta: 13 de marzo de 2016].

Salgado de Snyder, Nelly, Tonatiuh González-Vázquez, Berenice Jáuregui Ortiz y Pastor Bonilla Fernánadez, 2005, «No hacen viejos los años sino los daños: envejecimiento y salud en varones rurales» Salud Pública de México, 47(4), pp. 294-302, <www. scielo.org.mx/scielo.php?script=sci_arttext\&pid=S003636342005000400007> [consulta: 13 de marzo de 2016)]. 
Scott, Joan, 2001, «Experiencia», La Ventana, 13, pp. 42-73.

Suñol Salas, Rosa 2001, «Entrevista al profesor Avedis Donabedian», Revista Calidad Asistencial, suplemento 16(1), pp. 8-9, <www.fadq.org/wp-content/uploads/2016/02/ Monografico-Avedis-1parte.pdf > [consulta: 12 de febrero de 2016].

Valdez, Elba Abril, Rosario Román, María José Cubillas, 2005, "Análisis de la oferta de servicios para adultos Mayores y sus necesidades manifiestas», Psicología y Salud, 15(1), pp. 127-133.

Wong, Rebeca, Mónica Espinoza y Alberto Palloni 2007, «Adultos mayores en contexto socioeconómico amplio: salud y envejecimiento», Revista Salud Pública de México, 49(4), pp. 436-447, <www.scielo.org.mx/pdf/spm/v49s4/v49s4a02.pdf> [consulta: 12 de marzo de 2016].

World Health Organization (WHO), 2013, «Health in the post-2015 development agenda: need for a social determinants of health approach», Joint statement of the UN Platform on Social Determinants of Health, <www.worldwewant2015.org/es/ node/300184> [consulta: 14 de diciembre de 2015].

Yumi, Katia y María Lucía Magalhães, 2007, «La polisemia en el concepto de calidad en la evaluación de programas y servicios de salud: rescatando la subjetividad», en María Lucía Magalhães y Francisco Mercado (Org.), Investigación Cualitativa en los Servicios de Salud, Guadalajara, Lugar, pp. 63-79.

Fecha de recepción: 24 de noviembre de 2015

Fecha de aceptación: 4 de abril de 2016 